\title{
Expression and prognostic value of PD-L1 in non-schistosoma-associated urinary bladder squamous cell carcinoma
}

\author{
Zhenhua Liu ${ }^{1 \#}$, Yisen Meng' ${ }^{1 \#}$, Yudong Cao ${ }^{2 \#}$, Yuke Chen ${ }^{1}$, Yu Fan ${ }^{1}$, Shaobo Li $^{3}$, Qun He ${ }^{1}$, Shiliang Wu ${ }^{1}$, \\ Wei Yu ${ }^{1}$, Jie Jin ${ }^{1}$
}

${ }^{1}$ Department of Urology, Peking University First Hospital and Institute of Urology, National Research Center for Genitourinary Oncology, Beijing 100034, China; ${ }^{2}$ Key Laboratory of Carcinogenesis and Translational Research (Ministry of Education/Beijing), Department of Urology, Peking University Cancer Hospital \& Institute, Beijing 100142, China; ${ }^{3}$ School of Basic Medicine, Fudan University, Shanghai 200032, China

Contributions: (I) Conception and design: W Yu, J Jin; (II) Administrative support: SL Wu, Q He; (III) Provision of study materials or patients: Y Cao, Z Liu; (IV) Collection and assembly of data: Y Fan; (V) Data analysis and interpretation: Y Chen, S Li, Y Meng; (VI) Manuscript writing: All authors; (VII) Final approval of manuscript: All authors.

\#These authors contributed equally to this work.

Correspondence to: Jie Jin, MD, PhD. Department of Urology, National Urological Cancer Center, Peking University First Hospital and Institute of Urology, Peking University, 8 Xishiku Street, Xicheng District, Beijing 100034, China. Email: jinjie@vip.163.com; Wei Yu, MD. Department of Urology, National Urological Cancer Center, Peking University First Hospital and Institute of Urology, Peking University, 8 Xishiku Street, Xicheng District, Beijing 100034, China. Email: yuweif@126.com.

Background: Non-schistosoma-associated urinary bladder squamous cell carcinoma (SqCC) has low incidence and is associated with chronic inflammation. Due to its unique etiology and pathology, expression of programmed cell death ligand 1 (PD-L1) in SqCC could be different from that of urothelial carcinoma, which may contribute to different responses to immunotherapy. In this study, we intended to explore the expression profile and prognostic value of PD-L1 in non-schistosoma-associated urinary bladder SqCC under the consideration of tumor-infiltrating lymphocytes' (TILs) density.

Methods: We conducted a retrospective study to review 604 bladder cancer patients who received radical cystectomy (RC) from 2009 to 2013 in Peking University First Hospital. We enrolled 67 bladder SqCC patients in total, including pure $\mathrm{SqCC}(\mathrm{n}=19)$ and mixed $\mathrm{SqCC}(\mathrm{n}=48$, with urothelial carcinoma). PD-L1 protein expression and TILs density were evaluated by immunohistochemistry.

Results: Nine female and 58 male patients (median age 67.4 years) were enrolled in the present study. There were 15 stage T1-2 patients and 52 stage T3-4 patients. 27 patients had N1-2 lymph node metastasis. Overall, 61.2\% cases were PD-L1-positive. Dense TILs coincided with higher PD-L1 expression rate. Median survival time of $\mathrm{PD}-\mathrm{L} 1$ positive cases was significantly higher than negative cases $(\mathrm{P}=0.026)$. During multivariate analysis, positive PD-L1 expression and dense TILs were independent protective factors affecting overall survival (OS, PD-L1: $\mathrm{P}=0.022$; TILs: $\mathrm{P}=0.010)$ and progression free survival (PFS, PD-L1: $\mathrm{P}=0.018$; TILs: $\mathrm{P}=0.009)$.

Conclusions: PD-L1 expression and dense TILs were frequently detected in urinary bladder SqCC tumors. Positive PD-L1 expression and dense TILs were correlated with better survival outcomes in nonschistosoma-associated urinary bladder SqCC. The immunotherapy targeting PD-L1 might be helpful to bladder SqCC patients.

Keywords: Bladder cancer; squamous cell carcinoma (SqCC); tumor-infiltrating lymphocytes (TILs); programmed death ligand-1; survival

Submitted Oct 01, 2019. Accepted for publication Jan 17, 2020.

doi: $10.21037 /$ tau.2020.02.12

View this article at: http://dx.doi.org/10.21037/tau.2020.02.12 


\section{Introduction}

Bladder cancer represents the most common malignant neoplasm of the urinary tract. Bladder cancer is histopathologically heterogeneous and comprises urothelial carcinoma (UC, more than 90\%), squamous cell carcinoma (SqCC), adenocarcinoma and neuroendocrine tumors (1). Accounting for less than $5 \%$ of all bladder cancers, SqCC is of low incidence (2), with limited published data. Although surgery is the standard therapy, bladder SqCC is an independent predictor of worse cancer-specific survival (CSS) and is associated with poor responses to chemotherapy and radiotherapy (3-5).

Bladder SqCC is closely linked to chronic inflammation $(6,7)$. The primary role of inflammatory cells within the tumor micro-environment (e.g., Tumor-infiltrating lymphocytes, TILs) is to distinguish malignant cells (8). The programmed cell death ligand-1 (PD-L1) pathway has distinct effects in immune regulation of anti-tumor hosts. By binding to its receptor in the micro-environment of TILs, PD-L1 may result in suppression of immune cells $(8,9)$. PD-L1 expression, identified by immunohistochemical staining, could be an independent prognostic marker (10).

PD-L1 has been shown to be expressed in approximately $15-35 \%$ of urothelial carcinomas obtained from patients' postcystectomy (11). In contrast, PD-L1 expression in urinary bladder SqCC was reported as $64.7 \%$ in Udager et al.'s study (3), and as 66.9\% in Owyong et al.'s study (12). However, $80 \%$ patients in Owyong et al.'s study have schistosomiasis associated $\mathrm{SqCC}$, and the schistosomiasis associated SqCCs are different from non-schistosoma-associated ones in epidemiology, pathogenesis and prognosis (12).

Since 2016, five immune checkpoint inhibitors (ICIs) have been introduced for metastatic urinary bladder carcinoma (mUBC) as second-line treatment of post platinum-based chemotherapy or for cisplatin-ineligible patients (13). However, only 20-30\% patients with mUBC (metastatic urothelial bladder carcinoma) achieved a partial or complete response to immune-checkpoint inhibitors, which could be interpreted by relatively lower expression of PD-L1 (15-35\%) in urothelial carcinoma (14). The etiology and pathogenesis of SqCC are closely related to inflammation, and the poor prognosis requires special attention. Therefore, it is meaningful to study the expression of PD-L1 in SqCC, which could also provide basic/experimental evidence for clinical immunotherapy studies. In the current study, we aimed to explore the expression and prognostic value of PD-L1 in non- schistosoma-associated urinary bladder SqCC under the consideration of TILs density.

\section{Methods}

\section{Patient selection}

We enrolled 604 muscle-invasive bladder cancer patients who underwent radical cystectomy (RC) from January 2009 to December 2013 in our center. A total of sixty-seven cases were pathologically diagnosed as non-schistosomaassociated bladder SqCC, including pure SqCC $(n=19)$ and mixed SqCC ( $\mathrm{n}=48$, with UC) (15). Patients were excluded if they had any of the following conditions: (I) patients with previously diagnosed cancers or autoimmune diseases; (II) patients with incomplete clinical data and unavailable cystectomy specimens; (III) patients who underwent neoadjuvant or adjuvant chemotherapy and/ or radiation therapy; (IV) patients with positive surgical margin status; (V) patients who were lost to follow-up. The research was approved by the Ethics Committee, Peking University First Hospital. Informed consents were obtained from all participants in the study. Clinical and pathological information was retrospectively collected from the medical record library database. The pTNM staging was based on the 7th IUCC/AJCC recommendations.

\section{Cystectomy specimens}

Formalin-fixed paraffin-embedded surgical specimens were stained using hematoxylin and eosin (H\&E). The TILs density was marked as low (scattered and rare infiltration of lymphocytes) or high (dense infiltration of lymphocytes) (16) (Figure 1).

The paraffin-embedded carcinoma tissue was utilized for immunohistochemistry (IHC). Obtained from paraffinembedded cystectomy specimens, 3 - $\mu$ m-thick sections were utilized for the determination of PD-L1 expression. The slides were incubated with a primary PD-L1 antibody [Trade name: PD-L1 (E1L3N $\left.{ }^{\circledR}\right) \mathrm{XP}^{\circledR}$; Rabbit; Dilution: 1:200; Corp: Cell Signaling Technology, USA] (17). Then a secondary antibody was used [Trade name: R.T.U Biotinylated Goat Anti-Rabbit IgG Antibody (Readyto-Use); Corp: Vector, USA] (17). The slides were then image developed with 3,3'-diaminobenzidine (DAB) kit (VECTASTAIN ${ }^{\circledR}$ ABC kits; Corp: Vector, USA) (17).

In the tumor microenvironment, we described PDL1 expression as the percentage of cells showing immune 

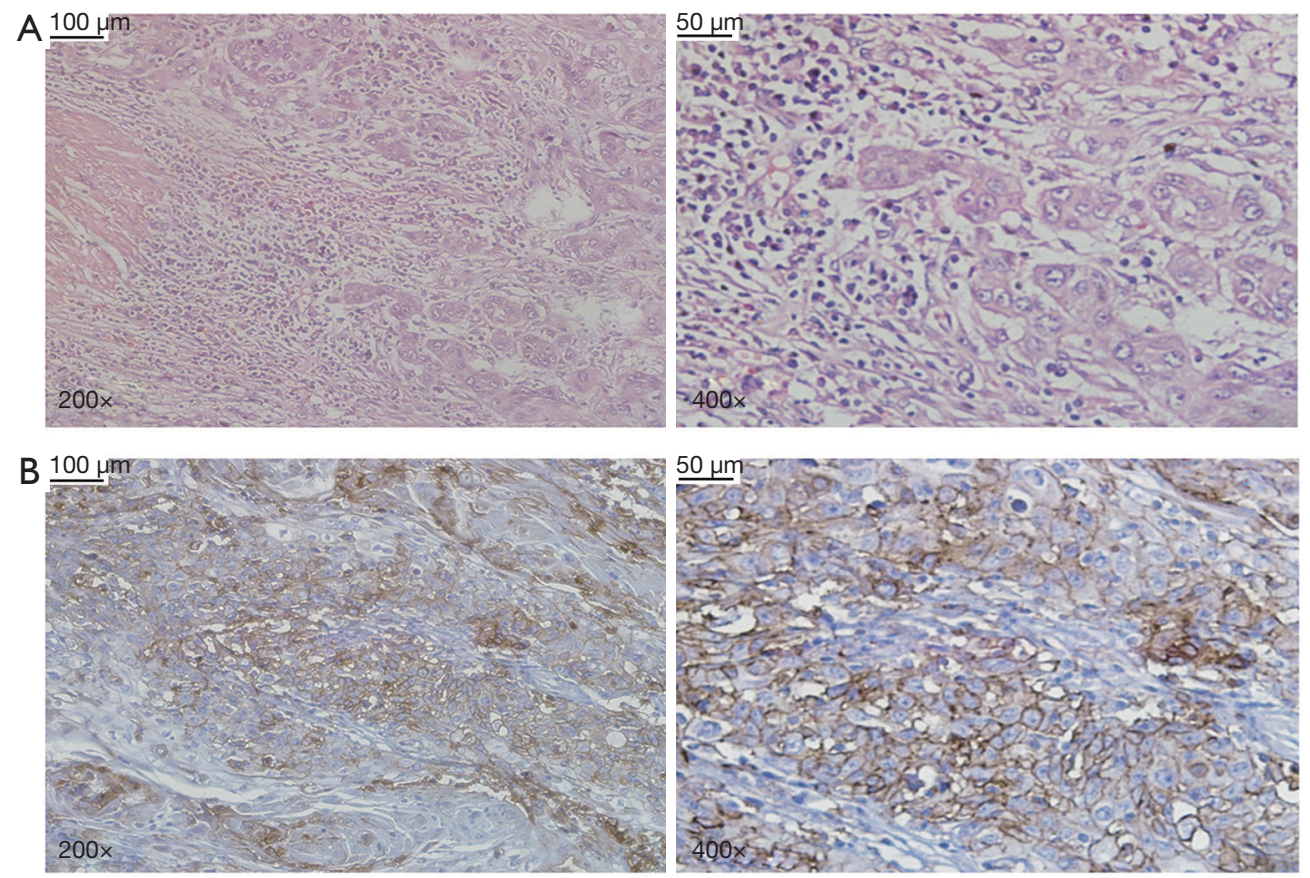

Figure 1 Immunohistochemistry (IHC) staining of bladder squamous cell carcinoma (SqCC). (A) Dense tumor-infiltrating lymphocytes (TILs). (B) expression of PD-L1 on bladder SqCC.

reactivity on the cell membrane (Figure 1). Whole areas were surveyed microscopically at 40 magnifications to identify focally stained regions. In cases of multiple areas of focal staining, three randomly selected areas were scored. Percentage of PD-L1 positive cells among total cells within focally stained regions was measured. Positive expression of PD-L1 was described as $>5 \%$ membranous staining $(18,19)$. Two experienced observers independently graded the staining with Image-Pro software (Corp: Media Cybernetics, Version $7^{\text {th }}$; USA) (17). When a discrepancy occurred, a third pathologist would examine the slides and determine the final score.

\section{Statistical analysis}

SPSS software (Corp: IBM, Version $20^{\text {th }}$; USA) was used in the statistical analysis. Student's $t$ test was used to compare groups, and Fisher's test to determine differences (17). We used Kaplan-Meier (K-M) method to estimate overall survival (OS) as well as progression-free survival (PFS) (20). We utilized Cox regression models to perform the univariate and multivariable survival analysis. All the statistics were two-sided, and statistically significant was considered at $\mathrm{P}$ value $<0.05$.

\section{Results}

\section{Demographic and clinical features}

Table 1 showed the patients' clinical characteristics as well as the demographic features. Generally, patients (median age 67.4 years) including 9 women and 58 men were enrolled in the study. There were 15 stage T1-2 patients and 52 stage T3-4 patients. There were 40 patients without lymph node metastasis and 27 patients with N1-2 lymph node metastasis. According to IHC, PD-L1 was expressed in $41 / 67$ cases $(61.2 \%)$. The expression of PD-L1 in the group with high TILs density $(29 / 37,78.4 \%)$ was higher than group with low TILs density (12/30, 40.0\%, $\mathrm{P}=0.002)$. In addition, PD-L1 expression was independent of tumor grade, $\mathrm{T}$ stage and $\mathrm{N}$ stage.

\section{Univariate analysis of survival outcomes}

The range of follow-up period was 2 to 79 months (median: 25 months). Thirty-nine out of 67 patients (58.2\%) developed specific recurrence or mortality of bladder cancer, and the rate of OS was $56.7 \%$ (38/67). According to $\mathrm{K}-\mathrm{M}$ curves (shown in Figure 2), patients with positive 
Table 1 Demographic and clinical characteristics of the study cohort

\begin{tabular}{|c|c|c|c|c|}
\hline Variables & Total, $\mathrm{N}=67$ & PD-L1 negative, $\mathrm{N}=26$ & PD-L1 positive, $\mathrm{N}=41$ & $P$ value \\
\hline Male & $58(86.6)$ & $23(39.7)$ & $35(60.3)$ & 0.511 \\
\hline Female & $9(13.4)$ & $3(33.3)$ & $6(66.7)$ & \\
\hline Age (years), median (range) & $68.0(42.0-88.0)$ & $64.5(49.0-88.0)$ & $71.00(42.0-85.0)$ & 0.100 \\
\hline Pure & $19(28.4)$ & $6(31.6)$ & $13(68.4)$ & 0.580 \\
\hline Mixed & $48(71.6)$ & $20(41.7)$ & $28(58.3)$ & \\
\hline \multicolumn{5}{|l|}{ Grade, n (\%) } \\
\hline G2 & $4(6.0)$ & $1(25.0)$ & $3(75.0)$ & 0.494 \\
\hline $\mathrm{T} 1-\mathrm{T} 2$ & $15(22.4)$ & $3(20.0)$ & $12(80.0)$ & 0.134 \\
\hline T3-T4 & $52(77.6)$ & $23(44.2)$ & $29(55.8)$ & \\
\hline \multicolumn{5}{|l|}{ N staging, n (\%) } \\
\hline No & $40(59.7)$ & $12(30.0)$ & $28(70.0)$ & 0.081 \\
\hline $\mathrm{N} 1-\mathrm{N} 2$ & $27(40.3)$ & $14(51.9)$ & $13(48.1)$ & \\
\hline \multicolumn{5}{|l|}{ TILs, n (\%) } \\
\hline Low & $30(44.8)$ & $18(60.0)$ & $12(40.0)$ & $0.002^{*}$ \\
\hline High & $37(55.2)$ & $8(21.6)$ & $29(78.4)$ & \\
\hline
\end{tabular}

PD-L1, programmed cell death-ligand 1; SqCC, squamous cell carcinoma; TILs, tumor-infiltrating lymphocytes, Mixed=SqCC with urothelial carcinoma. * $\mathrm{P}<0.05$.

PD-L1 expression had an OS rate of $53.7 \%$ (22/41), and the average survival time was 52 months $[95 \%$ confidence interval (CI): 33.1-70.9 months], which was obviously longer than PD-L1 negative patients (7 of 26, rate: $26.9 \%$; average survival time: 18 months) (95\% CI: 9.3-26.7 months, $\mathrm{P}=0.026$ ) (21). OS rate of dense TILs cases was obviously better than sparse TILs cases [19/37 (51.4\%) vs. 10/30 (33.3\%), $\mathrm{P}=0.044]$. Twenty-two in 41 (rate: 53.7\%) PD-L1 positive cases and 6 in 26 (rate: $23.1 \%$ ) PD-L1 negative cases did not suffer from disease progression $(\mathrm{P}=0.012)$. We did not observe a significant difference in disease progression between patients with high and low density of TILs.

We further examined the influence of PD-L1 expression on survival outcomes in combination with the density of TILs (Figure 3). According to Figure 3, positive PD-L1 combined with dense TILs cases had the highest $\mathrm{OS}$ rate $(\mathrm{P}=0.021)$ as well as PFS rate $(\mathrm{P}=0.028)(21)$. Nevertheless, we could not find a significant difference in OS or PFS between cases with PD-L1 positive tumors with a low density of TILs, PD-L1 negative tumors with a high density of TILs, and PD-L1 negative tumors with a low density of TILs.

\section{Multivariate analysis of survival outcomes}

We utilized Cox regression models for analyzing the OS and PFS (Table 2) risk factors. Positive PD-L1 expression together with high density of TILs turned out to be independent protective factors for OS [expression of $\mathrm{PD}$ L1: hazard ratio $(\mathrm{HR})=0.402, \mathrm{P}=0.022$; high density of TILs: HR $=0.344, \mathrm{P}=0.010$ ] as well as PFS (expression of PD-L1: HR $=0.394, \mathrm{P}=0.018$; high density of TILs: HR $=0.347, \mathrm{P}=0.009$ ) (21). Compared with patients with mixed SqCC, patients with pure SqCC exhibited worse OS (HR $=2.361, \mathrm{P}=0.038)$. What's more, lymph node metastasis was independently linked with worse OS and PFS. 

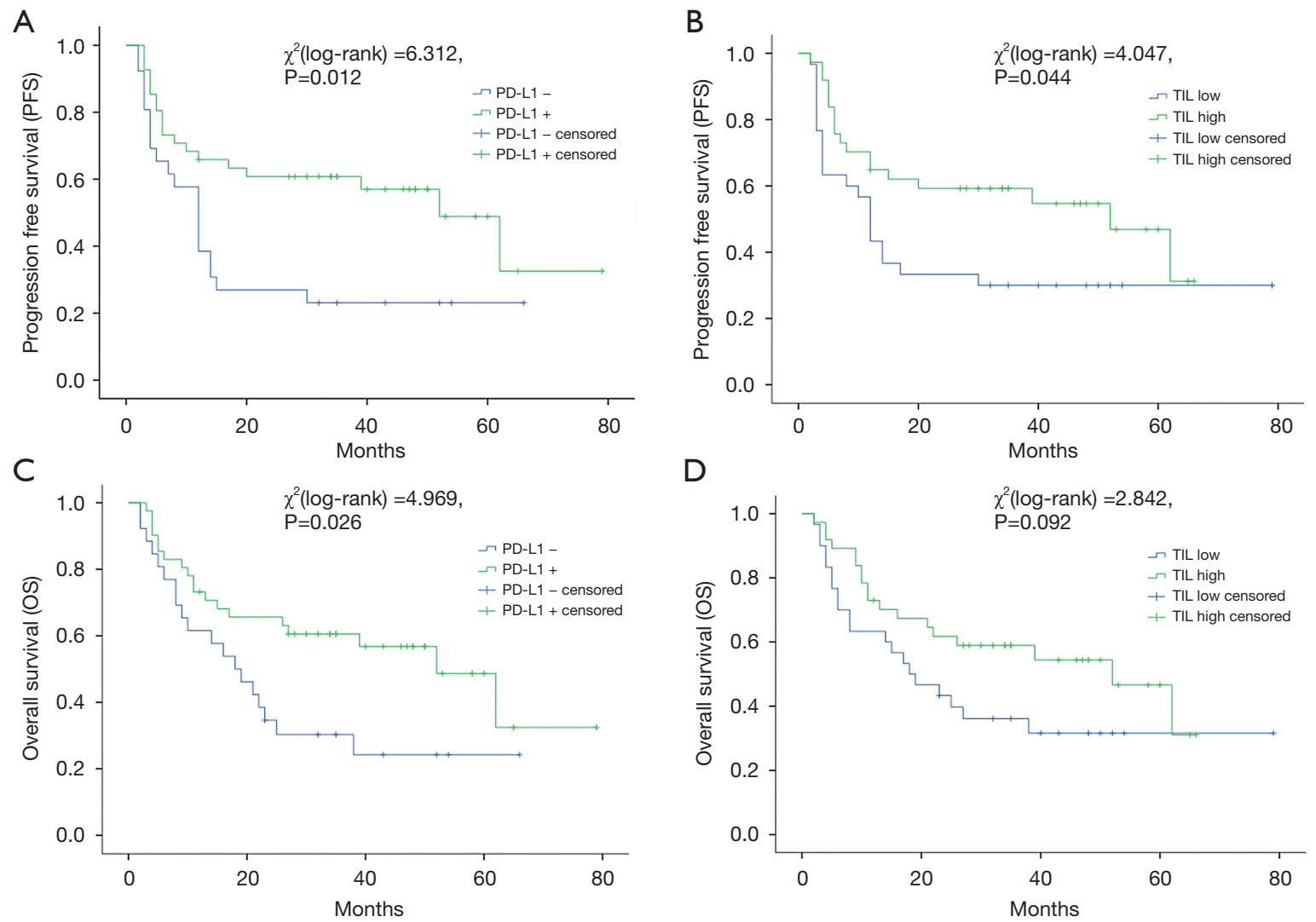

Figure 2 Kaplan-Meier curves of the progression-free survival (PFS) and overall survival (OS) for the whole study cohort. Kaplan-Meier curves of the PFS for the whole study cohort: (A) PD-L1 positive cases versus PD-L1 negative cases; (B) cases with a high density of tumorinfiltrating lymphocytes (TILs) versus cases with low density of TILs. Kaplan-Meier curves of the OS for the whole study cohort: (C) PDL1 positive cases versus PD-L1 negative cases; (D) cases with a high density of TILs versus cases with low density of TILs.
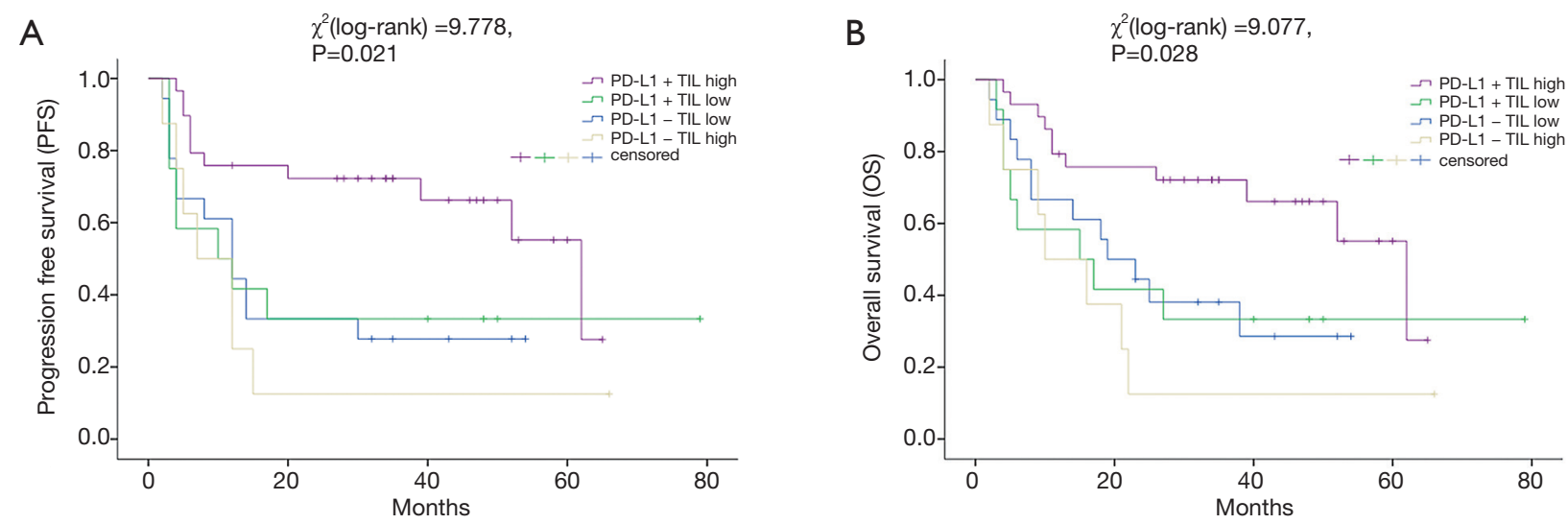

Figure 3 The influence of the PD-L1 expression on survival outcomes in combination with the density of tumor-infiltrating lymphocytes (TILs): Kaplan-Meier curves of the (A) progression-free survival (PFS) and (B) overall survival (OS) for the whole study cohort. 
Table 2 Multivariate analysis of prognostic factors for progression-free survival and overall survival

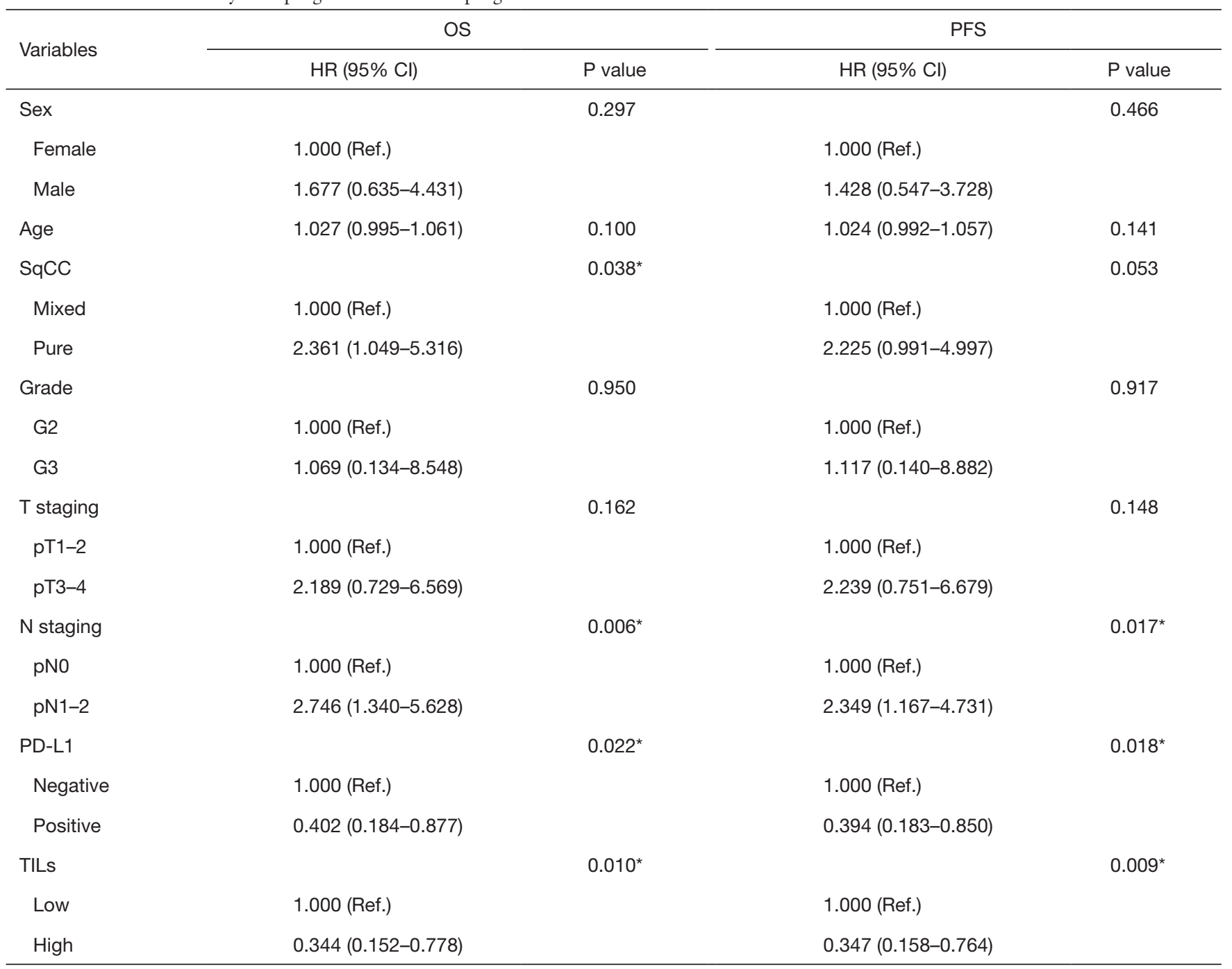

${ }^{*}, \mathrm{P}<0.05$. PD-L1, programmed cell death-ligand 1; SqCC, squamous cell carcinoma; TILs, tumor-infiltrating lymphocytes; Mixed, SqCC with urothelial carcinoma; PFS, progression-free survival, OS, overall survival; HR, hazard ratio.

\section{Discussion}

\section{PD-L1 expression and TILs in urinary bladder SqCC tumors}

Non-schistosoma associated urinary bladder SqCC is of low incidence with poor long-term survival outcomes (3-5). Different from other carcinomas, non-schistosoma associated urinary bladder SqCC is more frequent in patients with chronic inflammation, urinary tract calculi or chronic bladder outlet obstruction (22). In other organs' SqCC, like cervical SqCC, pulmonary SqCC as well as penile $\mathrm{SqCC}$, the positive $\mathrm{PD}-\mathrm{L} 1$ expression was reported to be higher compared with other subtypes of carcinomas (e.g., PD-L1 expression in lung cancer: $\mathrm{SqCC}$ is $52 \%$, whereas adenocarcinoma is $17 \%)(18,23-25)$.

To the best of our knowledge, this is the first study to report the expression as well as prognostic significance of PD-L1 and the tumor microenvironment in nonschistosoma-associated urinary bladder SqCC. The expression of PD-L1 with a $1 \%$ or $5 \%$ cut-off for positivity has been investigated in bladder cancer (26). The incidence of PD-L1 expression in post-cystectomy specimens of urothelial carcinomas was reported to be approximately 15 $35 \%(11,19,26)$. The expression of PD-L1 in mBUC may be related to response to immunotherapy (14). Previous studies have shown that PD-L1 is highly expressed in 
bladder SqCC. However, their results are limited because of small sample sizes [17 cases in Udager et al. (3)] or less representative as to schistosomiasis-associated SqCC [Owyong et al. (12)]. Therefore, it is important to study the expression of PD-L1 in non-schistosoma-associated bladder $\mathrm{SqCC}$, which could also provide important information for subsequent immunotherapy studies. In our study, 41 out of $67(61.2 \%)$ patients with urinary bladder SqCC had PD-L1 expression. Therefore, there appears to be a vital difference between PD-L1 expression in the major histological subtype of bladder cancer and bladder SqCC. The following reasons may explain that huge diversity. Bladder cancer is usually molecularly heterogeneous, particularly in the basal-squamous subtype (27). Nowadays, there is a tendency to view SqCC as a phenotype (the basalsquamous subtype) with common characteristics, namely, the expression of basal and stem-like markers (CD44, KRT5, KRT6A, KRT14) and SqCC markers (TGM1, DSC3, PI3), less frequent polysomy and genetic alterations and more frequent loss of chromosome $3 p$ (2). What's more, non-schistosoma associated bladder SqCC is more closely associated with chronic inflammation than other histological subtypes $(6,7,12)$, and might cause a general inflammatory response (28-30) as well as adaptive immune resistance with PD-L1 upregulation. Further, more studies at the molecular level are imperative.

\section{Prognostic value of PD-L1 expression and TILs in urinary bladder SqCC tumors}

Despite that some previous studies showed that positive PD-L1 expression was related to poor outcome of several malignancies (31), this matter remains controversial. Gabrielson et al. found that positive PD-L1 staining predicted a lower rate of recurrence as well as prolonged recurrencefree survival (RFS) in hepatocellular carcinoma (32). Schalper et al. reported PD-L1 mRNA expression was identified in nearly $60 \%$ of breast cancers and was associated with increased infiltration of TILs and improved RFS (33). Toyokawa et al. reported favorable disease-free survival was associated with PD-L1 expression in patients with surgically resected small-cell lung cancer (34).

Our results demonstrated that PD-L1 expression as well as high density of TILs were associated with better survival outcomes in non-schistosoma associated urinary bladder SqCC. This was consistent with Owyong et al.'s finding that PD-L1 expression was a favorable prognostic factor (in majority schistosomiasis associated bladder
SqCC) (12). The balance of the host's immune response and negative feedback inhibition could dominate outcome (35). The expression of PD-L1 in tumor microenvironment might reflect the presence of antigen-induced anti-tumor immune pressure mediated by TILs, and TILs recruited to the tumor microenvironment could still induce a partial anti-tumor effect $(8,36)$. Therefore, $\mathrm{PD}-\mathrm{L} 1$ positivity may signify tumors that have elicited an immune response, and are more likely to associate with better survival (37).

Our results suggest that bladder SqCC should be included in clinical trials to further observe whether survival benefits can be obtained. Our study has some limitations. As a retrospective study from a single medical center, selection bias was inevitable. Our findings need to be confirmed with larger samples and multi-center studies in the future.

\section{Conclusions}

PD-L1 expression and TILs were found in nonschistosoma-associated urinary bladder SqCC. PD-L1 expression profile was related to the density of TILs. In patients with non-schistosoma-associated urinary bladder SqCC, positive PD-L1 expression and dense TILs were associated with better survival outcomes.

\section{Acknowledgments}

We are grateful to all relevant physicians of Peking University First Hospital for allowing us to include their patients. The experimental data at the initial stage was reported in the International Conference on $33^{\text {rd }}$ Annual European Association of Urology (EAU) Congress (Copenhagen, 16-20 March 2018).

Funding: This study was supported by grants from the National Natural Science Foundation of China (Grant No. 81770755 \& 81570683 to J Jin) and supported by the Tibetian Natural Science Foundation (Grant No. XZ2017ZR-ZY019 to Y Meng).

\section{Footnote}

Conflicts of Interest: All authors have completed the ICMJE uniform disclosure form (available at http://dx.doi. org/10.21037/tau.2020.02.12). The authors have no conflicts of interest to declare.

Ethical Statement: The authors are accountable for all aspects of the work in ensuring that questions related 
to the accuracy or integrity of any part of the work are appropriately investigated and resolved. This study was approved by the institutional ethics committee of Peking University First Hospital \{approval number: 2016[1037]\}. Because it was a retrospective analysis of routine data, a waiver of wrote informed consent was granted from the ethics committee. Patient records or information were anonymous and de-identified prior to analysis.

Open Access Statement: This is an Open Access article distributed in accordance with the Creative Commons Attribution-NonCommercial-NoDerivs 4.0 International License (CC BY-NC-ND 4.0), which permits the noncommercial replication and distribution of the article with the strict proviso that no changes or edits are made and the original work is properly cited (including links to both the formal publication through the relevant DOI and the license). See: https://creativecommons.org/licenses/by-ncnd/4.0/.

\section{References}

1. Humphrey PA, Moch H, Cubilla AL, et al. The 2016 WHO Classification of Tumours of the Urinary System and Male Genital Organs-Part B: Prostate and Bladder Tumours. Eur Urol 2016;70:106-19.

2. Molitor M, Junker K, Eltze E, et al. Comparison of structural genetics of non-schistosoma-associated squamous cell carcinoma of the urinary bladder. Int J Clin Exp Pathol 2015;8:8143-58.

3. Udager AM, McDaniel AS, Hovelson DH, et al. Frequent PD-L1 Protein Expression and Molecular Correlates in Urinary Bladder Squamous Cell Carcinoma. Eur Urol 2018;74:529-31.

4. Scosyrev E, Ely BW, Messing EM, et al. Do mixed histological features affect survival benefit from neoadjuvant platinum-based combination chemotherapy in patients with locally advanced bladder cancer? A secondary analysis of Southwest Oncology Group-Directed Intergroup Study (S8710). BJU Int 2011;108:693-9.

5. Izard JP, Siemens DR, Mackillop WJ, et al. Outcomes of squamous histology in bladder cancer: a population-based study. Urol Oncol 2015;33:425.e7-13.

6. Youssef RF, Kapur P, Mosbah A, et al. Role of fibroblast growth factor in squamous cell carcinoma of the bladder: prognostic biomarker and potential therapeutic target. Urol Oncol 2015;33:111.e1-7.

7. Spradling K, Lotan Y, Shokeir A, et al. Lymphovascular invasion is associated with oncologic outcomes following radical cystectomy for squamous cell carcinoma of the urinary bladder. Urol Oncol 2016;34:417.e1-8.

8. Bardoli AD, Afshar M, Viney R, et al. The PD-1/PD$\mathrm{L} 1$ axis in the pathogenesis of urothelial bladder cancer and evaluating its potential as a therapeutic target. Future Oncol 2016;12:595-600.

9. Enkhbat T, Nishi M, Takasu C, et al. Programmed Cell Death Ligand 1 Expression Is an Independent Prognostic Factor in Colorectal Cancer. Anticancer Res 2018;38:3367-73.

10. Ragos V, Mastronikolis NS, Tsiambas E, et al. $\mathrm{PD} / \mathrm{L} 1$ in oral squamous cell carcinoma. J Buon 2018;23:835-6.

11. Baras AS, Drake C, Liu JJ, et al. The ratio of CD8 to Treg tumor-infiltrating lymphocytes is associated with response to cisplatin-based neoadjuvant chemotherapy in patients with muscle invasive urothelial carcinoma of the bladder. Oncoimmunology 2016;5:e1134412.

12. Owyong M, Lotan Y, Kapur P, et al. Expression and prognostic utility of PD-L1 in patients with squamous cell carcinoma of the bladder. Urol Oncol 2019;37:478-84.

13. Hsu FS, Su CH, Huang KH. A Comprehensive Review of US FDA-Approved Immune Checkpoint Inhibitors in Urothelial Carcinoma. J Immunol Res 2017;2017:6940546.

14. Ghate K, Amir E, Kuksis M, et al. PD-L1 expression and clinical outcomes in patients with advanced urothelial carcinoma treated with checkpoint inhibitors: A metaanalysis. Cancer Treat Rev 2019;76:51-6.

15. Wishahi M, Elganzoury H, Elhouly A, et al. Urothelial carcinoma of the urinary bladder mixed with squamous differentiation or squamous cell carcinoma in areas with schistosomiasis is showing high risk of recurrence and poor survival. J Egypt Soc Parasitol 2014;44:467-73.

16. Salgado R, Denkert C, Demaria S, et al. The evaluation of tumor-infiltrating lymphocytes (TILs) in breast cancer: recommendations by an International TILs Working Group 2014. Ann Oncol 2015;26:259-71.

17. Chen Y, Yu W, Yang Y, et al. Expression of programmed death ligand-1 on bladder tissues is detected in a clinically and histologically well-defined interstitial cystitis cohort. Neurourol Urodyn 2018;37:1396-404.

18. Ottenhof SR, Djajadiningrat RS, de Jong J, et al. Expression of Programmed Death Ligand 1 in Penile Cancer is of Prognostic Value and Associated with HPV Status. J Urol 2017;197:690-7.

19. Mukherji D, Jabbour MN, Saroufim M, et al. Programmed Death-Ligand 1 Expression in Muscle-Invasive Bladder Cancer Cystectomy Specimens and Lymph Node 
Metastasis: A Reliable Treatment Selection Biomarker?

Clin Genitourin Cancer 2016;14:183-7.

20. Grosso J, Horak CE, Inzunza D, et al. Association of tumor PD-L1 expression and immune biomarkers with clinical activity in patients (pts) with advanced solid tumors treated with nivolumab (anti-PD-1; BMS-936558; ONO4538). J Clin Oncol 2013;31:3016.

21. Chen Y, Cao Y, Yang Y, et al. The prognostic significance of tumour-infiltrating lymphocytes and programmed death ligandexpression in patients with squamous cell carcinoma of bladder. Eur Urol 2018;17:e137.

22. Lagwinski N, Thomas A, Stephenson AJ, et al. Squamous cell carcinoma of the bladder: a clinicopathologic analysis of 45 cases. Am J Surg Pathol 2007;31:1777-87.

23. Heineman TE, Widman A, Kuan EC, et al. The genetic landscape of programmed death ligand-1 (PD-L1) alterations in head and neck cancer. Laryngoscope Investig Otolaryngol 2017;2:99-103.

24. Müller T, Braun M, Dietrich D, et al. PD-L1: a novel prognostic biomarker in head and neck squamous cell carcinoma. Oncotarget 2017;8:52889-900.

25. Janzic U, Kern I, Janzic A, et al. PD-L1 Expression in Squamous-cell Carcinoma and Adenocarcinoma of the Lung. Radiol Oncol 2017;51:357-62.

26. Boussiotis VA. Molecular and Biochemical Aspects of the PD-1 Checkpoint Pathway. N Engl J Med 2016;375:1767-78.

27. Warrick JI, Sjodahl G, Kaag M, et al. Intratumoral Heterogeneity of Bladder Cancer by Molecular Subtypes and Histologic Variants. Eur Urol 2019;75:18-22.

28. Robertson AG, Kim J, Al-Ahmadie H, et al. Comprehensive Molecular Characterization of MuscleInvasive Bladder Cancer. Cell 2017;171:540-56.e25.

29. Choi W, Porten S, Kim S, et al. Identification of

Cite this article as: Liu Z, Meng Y, Cao Y, Chen Y, Fan Y, Li S, He Q, Wu S, Yu W, Jin J. Expression and prognostic value of PD-L1 in non-schistosoma-associated urinary bladder squamous cell carcinoma. Transl Androl Urol 2020;9(2):428-436. doi: $10.21037 /$ tau.2020.02.12 distinct basal and luminal subtypes of muscle-invasive bladder cancer with different sensitivities to frontline chemotherapy. Cancer Cell 2014;25:152-65.

30. Tan TZ, Rouanne M, Tan KT, et al. Molecular Subtypes of Urothelial Bladder Cancer: Results from a Meta-cohort Analysis of 2411 Tumors. Eur Urol 2019;75:423-32.

31. Takamori S, Toyokawa G, Okamoto I, et al. Clinical Significance of PD-L1 Expression in Brain Metastases from Non-small Cell Lung Cancer. Anticancer Res 2018;38:553-7.

32. Gabrielson A, Wu Y, Wang H, et al. Intratumoral CD3 and CD8 T-cell Densities Associated with Relapse-Free Survival in HCC. Cancer Immunol Res 2016;4:419-30.

33. Schalper KA, Velcheti V, Carvajal D, et al. In situ tumor PD-L1 mRNA expression is associated with increased TILs and better outcome in breast carcinomas. Clin Cancer Res 2014;20:2773-82.

34. Toyokawa G, Takada K, Haratake N, et al. Favorable Disease-free Survival Associated with Programmed Death Ligand 1 Expression in Patients with Surgically Resected Small-cell Lung Cancer. Anticancer Res 2016;36:4329-36.

35. Taube JM, Anders RA, Young GD, et al. Colocalization of inflammatory response with B7-h1 expression in human melanocytic lesions supports an adaptive resistance mechanism of immune escape. Sci Transl Med 2012;4:127ra37.

36. Liu CQ, Xu J, Zhou ZG, et al. Expression patterns of programmed death ligand 1 correlate with different microenvironments and patient prognosis in hepatocellular carcinoma. Br J Cancer 2018;119:80-8.

37. Davick JJ, Frierson HF, Smolkin M, et al. PD-L1 expression in tumor cells and the immunologic milieu of bladder carcinomas: a pathologic review of 165 cases. Hum Pathol 2018;81:184-91. 